
\title{
25 Research Soure \\ For young athletes undergoing ACL reconstruction, age may be key to surgical success
}

Sheena R. Black

Meghan Price

Colleen Wixted

Michael Heller

Lori Ann Asaro

Joseph Nguyen

Daniel W. Green

\section{Video Abstract}

Keywords: anterior cruciate ligament, $A C L$, reconstruction, revision, surgery, return to sport, pediatric, children, adolescents, skeletal age, knee ligament, sports medicine, age-related risk, skeletal maturity, all epiphyseal, partial transphyseal, complete transphyseal, growth disturbance, young athletes, growth failure

Posted Date: September 20th, 2019

DOI: https://doi.org/10.21203/rs.2.15021/v1

License: (9) This work is licensed under a Creative Commons Attribution 4.0 International License. Read Full License 


\section{Abstract}

Many factors influence recovery from surgical ACL reconstruction, and defining these is key to achieving the best outcomes. While the elements linked to surgical success in adult populations are well defined, they aren't so clear for younger patients. New research from the Hospital for Special Surgery in New York suggests that for these patients, age matters when it comes to protecting the knee. That conclusion stems from an evaluation of 324 athletes under 20 years of age who underwent ACL reconstruction following a sports injury. A key consideration when operating on this group is minimizing the risk of growth disturbances. Children and young adolescents haven't reached skeletal maturity, which has led to the development of age-specific surgical techniques. Researchers compared several of these approaches among groups divided based on skeletal age, school age distribution, surgical technique, and graft selection. Clinical follow-up was performed for at least 2 years. Intriguingly, the youngest and oldest participants achieved the same revision rate for reconstruction. But the middle group of athletes, composed of 8th and 9th graders, didn't fare as well, being nearly 3 times more likely to need additional surgery. They also returned to sports less often, and at lower performance levels, than the other age groups. So why are 8th and 9th graders more likely to experience poor outcomes after ACL surgery? Similar surgical approaches were used between middle and high schoolers with clearly different results, suggesting the surgery itself wasn't to blame. Graft choice also didn't seem to matter, as similar graft types were used for elementary and middle schoolers. The researchers speculate that age itself may be the key. Recovering 8th and 9th graders lose nearly a year of athletic activity during a critical time of skeletal growth, limiting their neuromuscular development as their skeleton matures. When they do return to sports, they must compete with high school athletes who have grown without losing time to an injury. Overall, it seems that some young patients undergoing ACL reconstruction aren't as prone to subsequent ACL injury as previously believed. Younger skeletally immature patients and older skeletally mature high school students are actually less likely to experience a new tear to the ACL than skeletally immature 8th and 9th graders. 